\title{
Long-Period Gratings in Highly Germanium-Doped, Single-Mode Optical Fibers for Sensing Applications
}

\author{
Sebastian Schlangen ${ }^{1, *}$, Kort Bremer ${ }^{1}$, Yulong Zheng ${ }^{1}$, Sebastian Böhm ${ }^{2}$, Michael Steinke ${ }^{2}$, \\ Felix Wellmann ${ }^{2}$, Jörg Neumann ${ }^{2}$, Bernhard Roth ${ }^{1}{ }^{10}$ and Ludger Overmeyer ${ }^{1}$ \\ 1 Hannover Centre for Optical Technologies, Leibniz Universität Hannover, Nienburger Str. 17, 30167 \\ Hannover, Germany; kort.bremer@hot.uni-hannover.de (K.B.); yulong.zheng@hot.uni-hannover.de (Y.Z.); \\ bernhard.roth@hot.uni-hannover.de (B.R.); ludger.overmeyer@ita.uni-hannover.de (L.O.) \\ 2 Laser Zentrum Hannover e.V., Hollerithallee 8, 30419 Hannover, Germany; s.boehm@lzh.de (S.B.); \\ m.steinke@lzh.de (M.S.); f.wellmann@lzh.de (F.W.); j.neumann@lzh.de (J.N.) \\ * Correspondence: sebastian.schlangen@hot.uni-hannover.de; Tel.: +49-511-762-17977
}

Received: 2 March 2018; Accepted: 25 April 2018; Published: 27 April 2018

\begin{abstract}
Long-period fiber gratings (LPGs) are well known for their sensitivity to external influences, which make them interesting for a large number of sensing applications. For these applications, fibers with a high numerical aperture (i.e., fibers with highly germanium $(\mathrm{Ge})$-doped fused silica fiber cores) are more attractive since they are intrinsically photosensitive, as well as less sensitive to bend- and microbend-induced light attenuations. In this work, we introduce a novel method to inscribe LPGs into highly Ge-doped, single-mode fibers. By tapering the optical fiber, and thus, tailoring the effective indices of the core and cladding modes, for the first time, an LPG was inscribed into such fibers using the amplitude mask technique and a $\mathrm{KrF}$ excimer laser. Based on this novel method, sensitive LPG-based fiber optic sensors only a few millimeters in length can be incorporated in bend-insensitive fibers for use in various monitoring applications. Moreover, by applying the described inscription method, the LPG spectrum can be influenced and tailored according to the specific demands of a particular application.
\end{abstract}

Keywords: long-period fiber grating; high NA fiber; amplitude mask; excimer laser; germanium-doped fiber; single-mode fiber

\section{Introduction}

Long-period fiber gratings (LPGs) are applied in order to couple light between core and co-propagating cladding modes in single-mode optical fibers. Introduced about two decades ago, LPGs have found many applications due to their characteristics and sensitivity. For example, external perturbations, due to mechanical stress, temperature, or refractive index changes in the surrounding medium, will change the grating period, as well as the effective refractive index of the modes. These effects can be utilized for the development of bend [1], torsion [2], temperature [3], and pressure [4], as well as refractive index sensors [4-6], which also find applications as biosensors due to special coatings of the fiber cladding to detect e.g. viruses [7] or bacteria [8].

The corresponding coupling wavelength $\lambda_{R}$ of the LPGs to couple light between the core mode and an individual cladding mode can be calculated as:

$$
\lambda_{\mathrm{R}}=\Lambda \cdot\left(\mathrm{n}_{\mathrm{eff}, \mathrm{Cr}}-\mathrm{n}_{\mathrm{eff}, \mathrm{Cl}}\right),
$$

where $\Lambda$ is the grating period and $n_{\text {eff,Cr }}$ and $n_{\text {eff,Cl }}$ are the effective refractive indices of the core and cladding mode, respectively. For the fabrication of LPGs, various manufacturing techniques 
are employed to realize a periodic modulation of the refractive index within the fiber core. Common methods are laser-based, point-by-point inscription systems utilizing, for example, $\mathrm{CO}_{2}$ infrared laser radiation operating either in continuous or in pulsed mode [9,10], or ultra-violet (UV) femtosecond laser radiation [11,12]. LPGs can also be realized by inducing mechanical stress or employing other physical modifications such as microbends [13], electric arc-discharges [14-16], or ion-implantation [17] whereby the optical spectrum can be optimized by etching processes [18]. One of the simplest and widely used methods for LPG fabrication is the amplitude mask (AM) technique [3,19-25]. Amplitude masks are periodic arrangements of rectangular and transparent slits that usually have a width of $\Lambda / 2$, and are separated by the grating period $\Lambda$. Furthermore, AMs are usually based on alternating chrome and chrome-less areas on a fused silica glass substrate [19], or metal sheets with rectangular recesses [26]. Compared to the point-by-point fabrication of LPGs, the main advantage of the AM-based manufacturing technique is that a simple, robust, and relatively low-cost optical setup is sufficient, and thus, this manufacturing technique is more suitable for mass production. The UV-induced LPG inscription is only reported for standard telecommunication fibers with numerical apertures (NAs) of 1.2-1.4, such as Fibercore PS1250/1500 (NAs 0.12-0.14), Nufern GF1 (NA 0.13), or Corning SMF-28 (NA 0.14), so far [4,12].

In terms of sensing applications, fibers with a high NA (i.e., fibers with highly Germanium (Ge)-doped fused silica fiber cores) are more attractive since they are less sensitive to bend/microbend-induced light attenuations, and are intrinsically photosensitive, and thus, are suitable for the fabrication of fiber grating structures using UV excimer laser light. The insensitivity to bend/microbend-induced light attenuation is particularly important when integrating optical fibers in functionalized textile structures that are applied for structural health monitoring (SHM) applications, for example [27]. However, when applying single-mode optical fibers with high NAs for use in LPG applications, it follows from Equation (1), that due to the large difference in the effective refractive indices between the core and cladding modes, small grating periods are required for the co-propagating IR coupling in a wavelength range between 1350 to 1600 . Since metal masks with small periods are difficult to fabricate, and the durability of chrome masks to high-power UV light exposure is limited, the manufacturing of LPGs in highly Ge-doped, single-mode fibers has not been reported so far.

In this work, we present for the first time a method to inscribe UV-induced LPGs in highly Ge-doped, single-mode optical fibers, which were previously subjected to a tapering process using the $\mathrm{AM}$ technique and $\mathrm{KrF}$ excimer laser light illumination. For the experiments, the highly Ge-doped, single-mode fiber SM1500 (4.2/125) from Fibercore (Southampton, UK) was applied, which had a high NA of 0.3 , and thus, ensured high photosensitivity and an inherent high-bending resistance. Different core and cladding diameters were investigated in order to determine their influence on the required grating period, as well as their impact on the resulting transmission spectrum of the LPGs. Moreover, the sensitivity of the spectrum to refractive index changes of the surrounding medium was exploited. The investigation began with optical simulations of the LPG inscription process, which were subsequently verified by experiments. This was then followed by the measurement of the influence induced by the refractive index change in the surroundings.

\section{Optical Simulations}

The optical simulation of the LPG inscription method was performed in RSoft (Synopsys, Inc., Mountain View, CA, USA) using the GratingMOD toolbox. The following parameters were applied for the Fibercore SM1500 single-mode fiber: refractive index of the fiber core $\mathrm{n}_{\mathrm{Cr}}=1.47$; refractive index of the cladding $\mathrm{n}_{\mathrm{Cl}}=1.44$; and core diameter $\mathrm{d}_{\mathrm{Cr}}=4 \mu \mathrm{m}$. For the $\mathrm{LPG}$, a grating length of $\mathrm{L}_{\mathrm{Gr}}=8 \mathrm{~mm}$, as well as a refractive index modulation of $\delta \mathrm{n}=10^{-3}$ within one period of the fiber grating, were assumed. The simulations were performed in a wavelength range between 1350 to $1600 \mathrm{~nm}$. For the simulation model, the refractive indices of the core and cladding were assumed to be constant, and thus, only waveguide dispersion effects were taken into account by the RSoft GratingMOD Toolbox. 


\subsection{Untreated Highly Ge-Doped, Single-Mode Optical Fiber}

At the beginning of the optical simulation, the required grating periods, as well as the resulting LPG spectrum for the untreated SM1500 single-mode fiber, were determined numerically. In this case, a cladding diameter of $\mathrm{d}_{\mathrm{Cl}}=125 \mu \mathrm{m}$, as well as a refractive index of the surrounding of $\mathrm{n}_{\mathrm{Sr}}=1$ were assumed. The nomenclature of the cladding modes was defined as $\mathrm{LP}_{\mathrm{m}, \mathrm{n}}$, where $\mathrm{m}=0$ and $\mathrm{n}=1,2$, $\ldots, 15$. In Figure $1 \mathrm{a}$, the calculated grating periods to couple light from the $\mathrm{LP}_{0,1}$ core mode to the $\mathrm{LP}_{0,1}-\mathrm{LP}_{0,15}$ cladding modes are illustrated. Moreover, in Figure $1 \mathrm{~b}$, the corresponding simulated normalized coupling coefficients are shown.
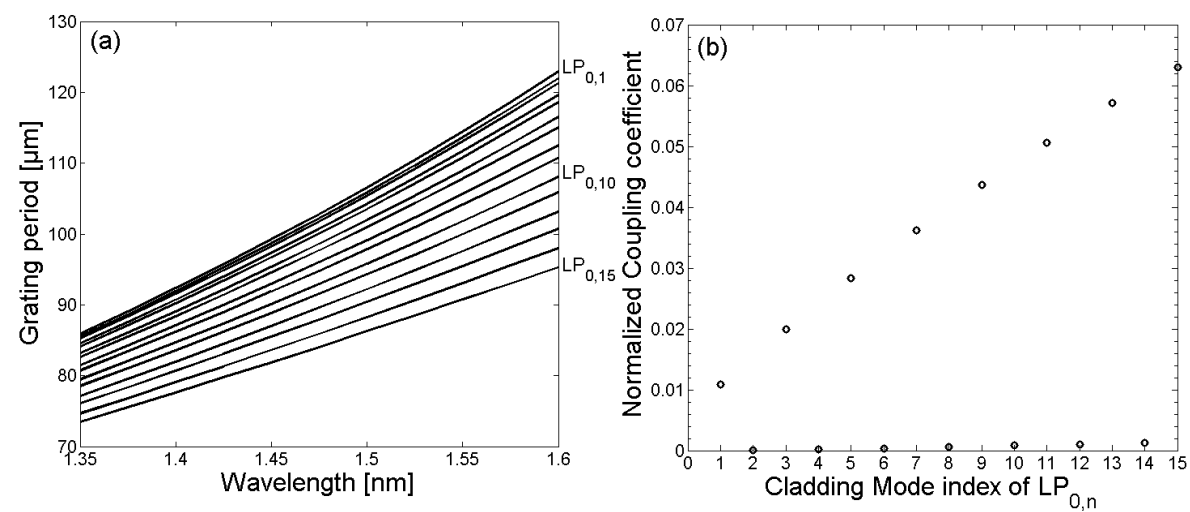

Figure 1. The relationship between grating period $\Lambda$ and wavelength $\lambda$ for coupling the core mode into the cladding modes $\mathrm{LP}_{0,1}-\mathrm{LP}_{0,15}$ is shown in (a), whereby the grating periods for coupling decrease steadily with increasing mode numbers. The corresponding normalized coupling coefficients are shown in (b).

Due to the relatively small core diameter, which is required in order to provide single-mode operation, resulting in a reduced overlap between the core and cladding mode field distributions, the normalized coupling coefficients between the $\mathrm{LP}_{0,1}$ core mode and the $\mathrm{LP}_{0, \mathrm{n}}$ cladding modes were relatively small. The resulting LPG transmission spectrum for an untreated SM1500 single-mode optical fiber with a grating period of $\Lambda=100 \mu \mathrm{m}$ is depicted in Figure 2. According to the calculated grating periods and the coupling coefficients, light was mainly coupled in a wavelength range of $1350 \mathrm{~nm}$ to $1600 \mathrm{~nm}$ between the $\mathrm{LP}_{01}$ core mode and the $\mathrm{LP}_{0,1}, \mathrm{LP}_{0,3}, \mathrm{LP}_{0,5}, \mathrm{LP}_{0,7}, \mathrm{LP}_{0,9}, \mathrm{LP}_{0,11}$, and $\mathrm{LP}_{0,13}$ cladding modes, respectively.

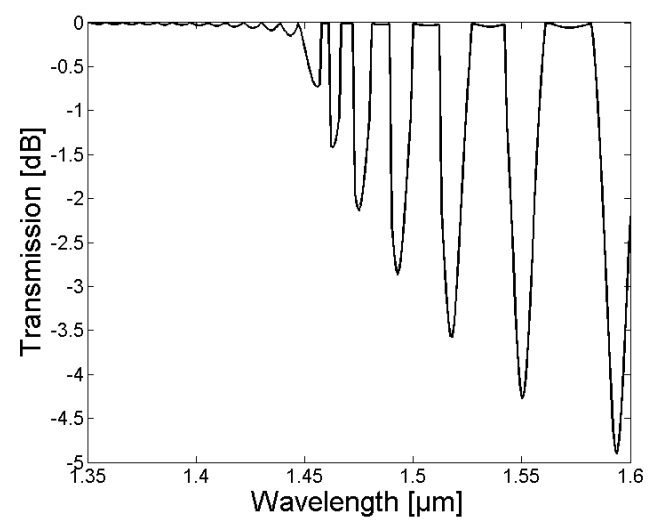

Figure 2. The resulting simulated long-period fiber grating (LPG) spectrum of an untreated SM1500 single-mode fiber with a grating period of $100 \mu \mathrm{m}$, a grating length of $8 \mathrm{~mm}$ and a refractive index modulation of $\delta \mathrm{n}=1 \times 10^{-3}$. 


\subsection{Tapered Highly Ge-Doped, Single-Mode Optical Fiber}

From Equation (1), it follows that when the effective refractive index of the core mode is reduced (i.e., when the difference between $\mathrm{n}_{\mathrm{eff}, \mathrm{C}}$ and $\mathrm{n}_{\mathrm{eff}, \mathrm{Cl}}$ decreases), the required grating period of the LPGs increase for a constant coupling wavelength. The effective refractive index of the fundamental core mode depends on the core diameter $\left(\mathrm{d}_{\mathrm{Cr}}\right)$, as well as the refractive index difference between the core and the cladding $(\Delta \mathrm{n})$. Since after the drawing process the refractive index profile of a fiber cannot be tailored, the only alternative is to reduce the core and cladding diameters (e.g., by tapering the fiber) in order to decrease the effective refractive index of the fiber core mode, and to achieve an LPG spectrum in the spectral range between the second and third optical window. This can be reached by applying grating periods of the order of a few hundred microns.

In order to verify this hypothesis, optical simulations of LPGs were performed when the SM1500 single-mode optical fiber was tapered. A parabolic model was applied to model the tapered optical fiber [28]. Based on this model, the diameter of the core $\mathrm{d}_{\mathrm{Cr}}$ and cladding $\mathrm{d}_{\mathrm{Cl}}$ can be calculated as [28]:

$$
\frac{\mathrm{D}_{\mathrm{f}}}{\mathrm{D}_{\mathrm{i}}}=\frac{\frac{3}{2}\left(4-\frac{1}{\Delta \mathrm{z}}\right)}{\left(1+\frac{1}{\Delta \mathrm{z}}\right)+5\left[\left(1+\frac{1}{\Delta \mathrm{z}}\right)\left(1-\frac{1}{5 \Delta \mathrm{z}}\right)\right]^{1 / 2}},
$$

where $D_{i}, D_{f}, 1$, and $\Delta z$ are the initial and final core and cladding diameter of the fiber before and after tapering, as well as the axial extension length during tapering, and the hot zone of the fiber (i.e., the length of the fiber that was heated during tapering), respectively. For the modelling of the shape of the tapered optical fiber, a hot zone with a length of $\Delta z=25 \mathrm{~mm}$ was assumed for the tapering process. To show how further modification of the LPG spectrum can be achieved by changing the relationship between core and cladding diameter, the optical simulation was performed with cladding diameters of $d_{C l}=125 \mu \mathrm{m}$ (Figures 3-5) and $d_{C l}=80 \mu \mathrm{m}$ (Figures 6-8), as the SM1500 fiber is commercially available for both cladding diameters.

In Figures 3 and 6, the calculated grating periods to couple light from the fundamental $\mathrm{LP}_{01}$ core mode to the $\mathrm{LP}_{0,1}-\mathrm{LP}_{0,10}$ cladding modes are shown, and the corresponding normalized coupling coefficients as a function of the fiber extension during the tapering process are given in Figures 4 and 7 . The LPG transmission spectra for a grating period of $\Lambda=370 \mu \mathrm{m}$ and an extension length $1=28 \mathrm{~mm}$ are illustrated in Figure 5 (initial cladding diameter of $125 \mu \mathrm{m}$ ) and Figure 8 (initial cladding diameter of80 $\mu \mathrm{m})$, respectively.

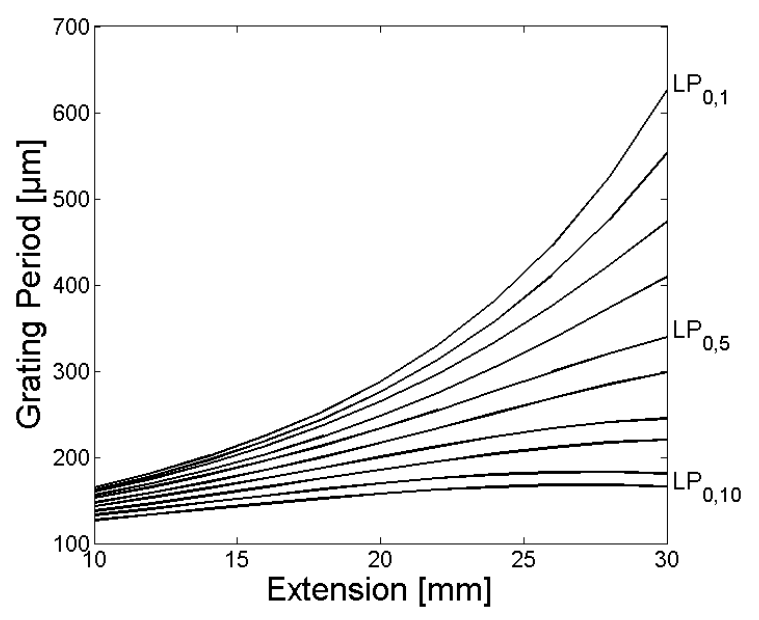

Figure 3. The calculated grating periods to couple light from the fundamental $\mathrm{LP}_{0,1}$ core mode to the $\mathrm{LP}_{0,1}-\mathrm{LP}_{0,10}$ cladding modes for the SM1500 fiber with a starting cladding diameter of $125 \mu \mathrm{m}$ are shown as a function of the fiber extension 1 , whereby the grating periods for coupling decrease steadily with increasing mode numbers. 

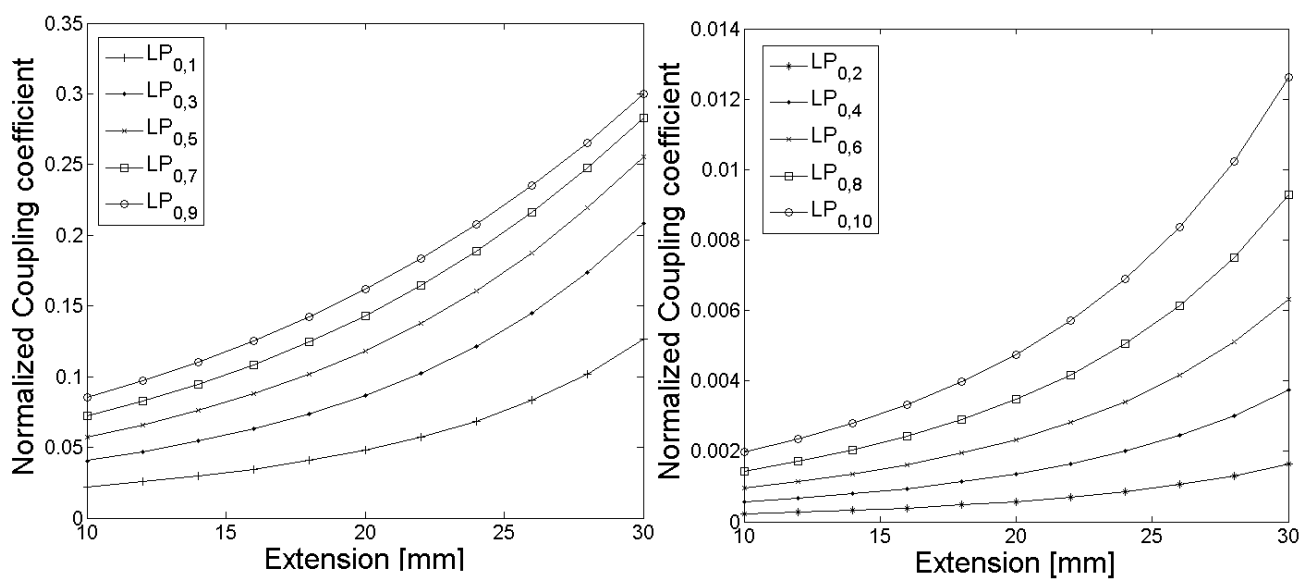

Figure 4. The normalized coupling coefficients as a function of the fiber extension 1 during the tapering process for coupling of light from the fundamental $\mathrm{LP}_{0,1}$ core mode to the $\mathrm{LP}_{0,1}-\mathrm{LP}_{0,10}$ cladding modes of the SM1500 fiber with a starting cladding diameter of $125 \mu \mathrm{m}$ are shown, whereby modes with odd mode numbers (left) have significant larger normalized coupling coefficients compared to the modes with even mode numbers (right).

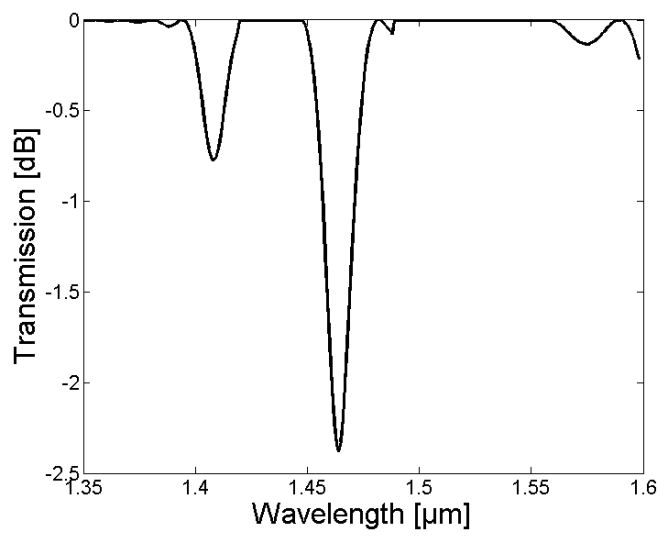

Figure 5. The resulting simulated LPG spectrum in the tapered SM1500 single-mode fiber with a starting cladding diameter of $125 \mu \mathrm{m}$, an extension of $28 \mathrm{~mm}$, a grating period of $370 \mu \mathrm{m}$, a grating length of $8 \mathrm{~mm}$, and a refractive index change within the grating of $\delta \mathrm{n}=1 \times 10^{-3}$.

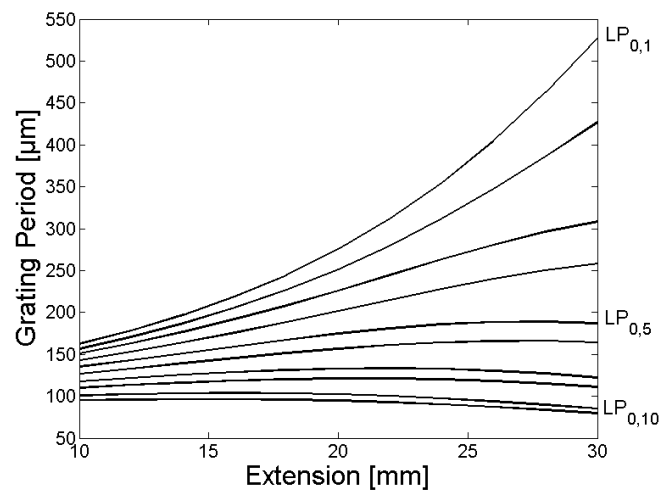

Figure 6. The calculated grating periods to couple light from the fundamental $\mathrm{LP}_{0,1}$ core mode to the $\mathrm{LP}_{0,1}-\mathrm{LP}_{0,10}$ cladding modes for the SM1500 fiber with a starting cladding diameter of $80 \mu \mathrm{m}$ are shown as function of the fiber extension, whereby the grating periods for coupling decrease steadily with increasing mode numbers. 

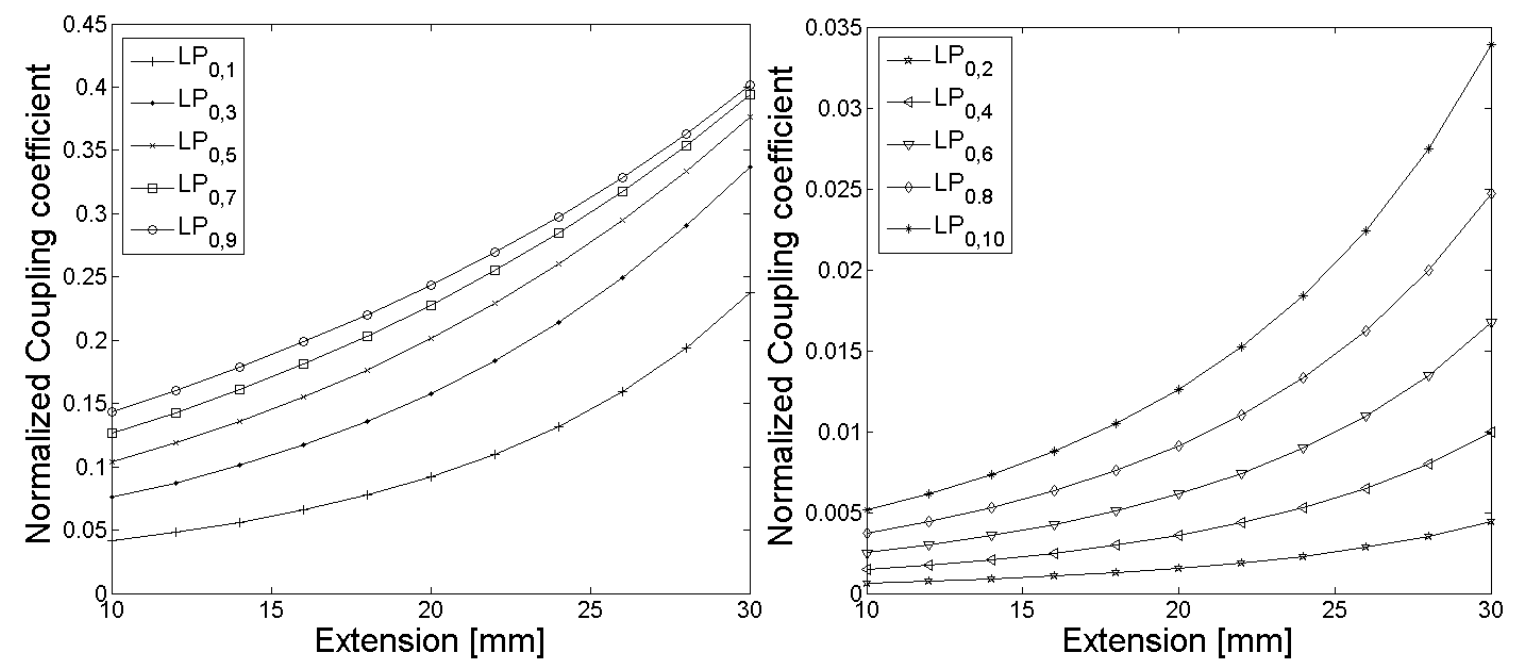

Figure 7. The normalized coupling coefficients as a function of the fiber extension during the tapering process for coupling of light from the fundamental $\mathrm{LP}_{0,1}$ core mode to the $\mathrm{LP}_{0,1}-\mathrm{LP}_{0,10}$ cladding modes for the SM1500 fiber with a starting cladding diameter of $80 \mu \mathrm{m}$ are shown, whereby modes with odd mode numbers (left) have significant larger normalized coupling coefficients compared to the modes with even mode numbers (right).

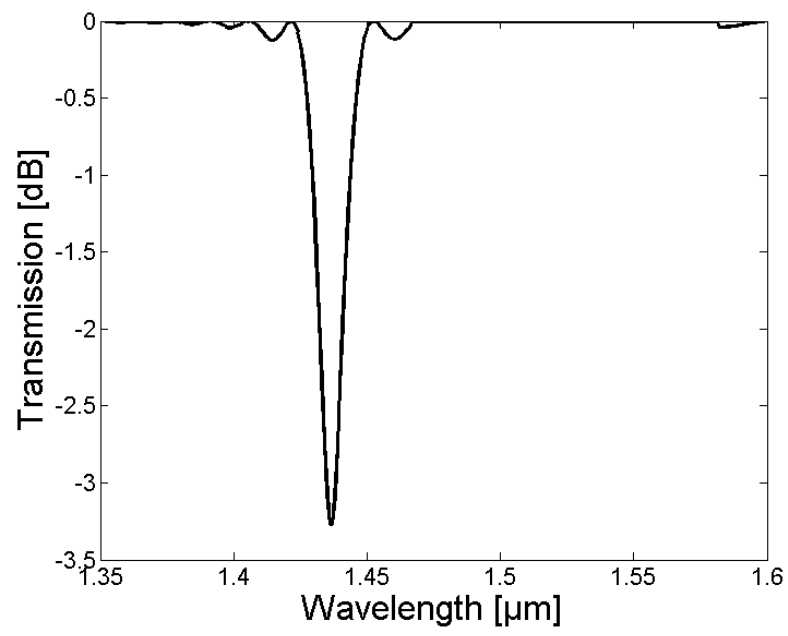

Figure 8. The resulting simulated LPG spectrum in the tapered SM1500 single-mode fiber, with a starting cladding diameter of $80 \mu \mathrm{m}$, an extension of $28 \mathrm{~mm}$, a grating period of $370 \mu \mathrm{m}$, a grating length of $8 \mathrm{~mm}$, and a refractive index change within the grating of $\delta \mathrm{n}=1 \times 10^{-3}$.

From Figures 3 and 6, it follows that the required grating period can be tailored by tapering the SM1500 single-mode optical fiber to couple light between the fundamental core mode and co-propagating cladding modes. Furthermore, the normalized coupling coefficient between the core and cladding modes, as well as the LPG transmission spectrum, can be optimized (only a single absorption dip appears in a relatively broad spectral range) when reducing the cladding diameter $\mathrm{d}_{\mathrm{Cl}}$ of the optical fiber prior to the tapering process as one can see in Figure 8.

\section{Fabrication of LPG in Tapered Highly Ge-Doped, Single-Mode Fiber}

In order to verify the optical simulation, an appropriate fabrication process was developed to inscribe LPGs in the highly Ge-doped, single-mode fiber SM1500. To show how the normalized coupling coefficient between the core and cladding modes, as well as the LPG transmission spectrum can further be optimized so that a single absorption dip appears in a relatively broad spectral range, 
an initial cladding diameter of $80 \mu \mathrm{m}$ prior to the tapering process was chosen for the experiments. Since, at this stage, only SM1500 fibers with a cladding diameter of $125 \mu \mathrm{m}$ were available, the cladding diameter was reduced at the beginning of the fabrication process from $\mathrm{d}_{\mathrm{Cl}}=125 \mu \mathrm{m}$ to $\mathrm{d}_{\mathrm{Cl}}=80 \mu \mathrm{m}$ $( \pm 1.25 \%)$ using hydrofluoric acid $(40 \%$ acid, $60 \%$ water). The fiber cladding diameter was etched in a Teflon chamber over a length of $55 \mathrm{~mm}$. Moreover, to obtain a constant etching rate, the temperature was kept constant at $21^{\circ} \mathrm{C}$. Following the etching process, the fiber was tapered. The tapering was done using a hydrogen burner with a melting zone of $\Delta z=25 \mathrm{~mm}$ and extending the fiber by $\mathrm{l}=28 \mathrm{~mm}$. After the tapering process, cladding and core diameters of $\mathrm{d}_{\mathrm{Cl}}=42 \mu \mathrm{m}( \pm 2.5 \%) \mu \mathrm{m}$ and $\mathrm{d}_{\mathrm{Cr}}=2.1 \mu \mathrm{m}$ $( \pm 2.5 \%) \mu \mathrm{m}$, respectively, were measured based on a total of 26 fiber tapers using a digital light microscope (Leitz Aristomet (ProMicron, Kirchheim am Neckar, Germany) with Variophot-Tubus and the CCD-Camera AxioCam MRc (Carl Zeiss, Jena, Germany)). The subsequent inscription of the LPG was done by UV light exposure using a KrF excimer laser (Atlex FBG (ATL Lasertechnik GmbH, Wermelskirchen, Germany)) at a wavelength of $248 \mathrm{~nm}$, a pulse energy of $7 \mathrm{~mJ}$, and a repetition rate of $80 \mathrm{~Hz}$. The LPG inscription setup is shown schematically in Figure 9.

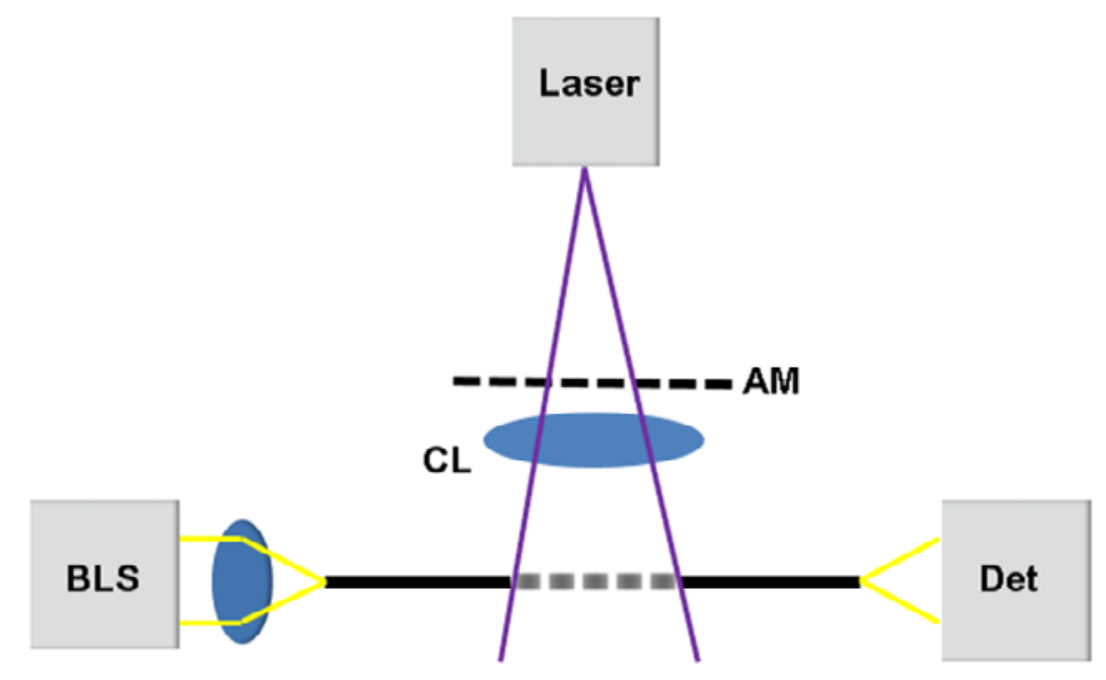

Figure 9. The setup for UV-induced LPG inscription and detection. The UV laser illuminates the amplitude mask (AM), and the generated LPG pattern is focused by a cylindrical lens (CL) in one transversal dimension onto the fiber. During the UV exposure, light is coupled into the fiber using a broadband light source (BLS), and the resulting LPG spectrum is detected the end of the fiber using an optical spectrum analyzer (OSA) as a detector (Det).

As illustrated in Figure 9, the laser beam first illuminates the AM in order to generate the periodic intensity pattern that was required for the LPG inscription. For inscription, chrome masks (Rose Fotomasken Milan, Milos \& Eva Rose GbR, Bergisch Gladbach, Germany) were applied. Behind the AM, the spatially modulated beam was focused horizontally using a plan-convex cylindrical lens (UVFS Plano-Convex Cylindrical Lens, $\mathrm{f}=10 \mathrm{~mm}$ (Thorlabs Inc., Newton, NJ, USA)) to obtain the required intensity density, and the tapered SM1500 fiber was placed in the focal point. The total inscription length of the experimental setup was $8 \mathrm{~mm}$. Since the laser beam was focused to a point behind the AM, the chrome mask was not destroyed during LPG inscription. Moreover, due to the $10 \mathrm{~mm}$ gap between the AM and the SM1500 fiber, as well as the vertical divergence of the laser beam, the spatially modulated intensity pattern changed from a perfect rectangular to a sinusoidal-like modulation. Furthermore, to record the LPG transmission spectrum during inscription, a broadband light source (BBS, stabilized fiber-coupled light source SLS201L/M (Thorlabs Inc., Newton, NJ, USA)), as well as an optical spectrum analyzer (OSA, Ando AQ6317B (Ando Electronic SRL, Tokyo, Japan)), were employed and bare fiber adapters were used to connect the fiber under test (i.e., tapered SM1500) 
to the BBS and OSA. The obtained spectrum was recorded in LabVIEW 2011 SP1 (National Instruments, Austin, TX, USA) and analyzed in MATLAB R2017b (The MathWorks, Inc., Natick, MA, USA).

\section{Experimental Results of the Inscription Process}

The simulations were experimentally verified by inscribing LPGs in the tapered SM1500 with grating periods of $370 \mu \mathrm{m}$ and $365 \mu \mathrm{m}$, respectively. The recorded LPG transmission spectra for both LPGs are illustrated in Figure 10. The exposure time to UV light from the KrF excimer laser was 30 min and 45 min for the LPGs with a grating period of 365 and 370, respectively.

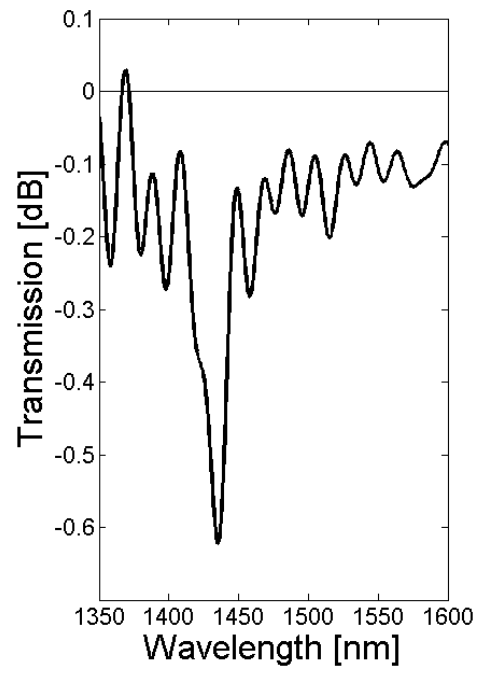

(a)

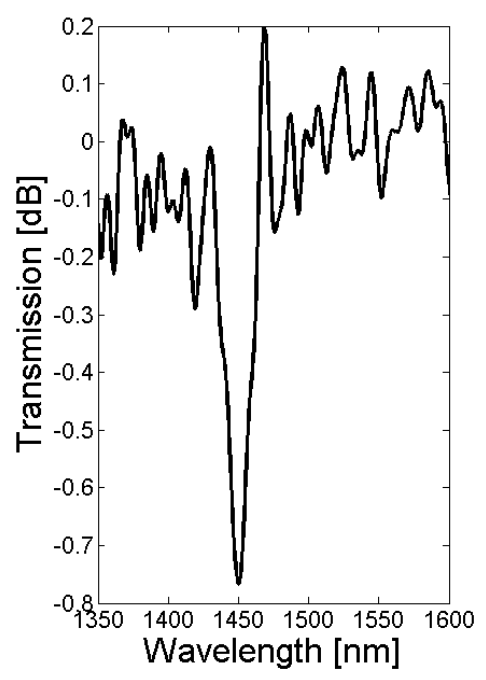

(b)

Figure 10. The measured LPG transmission spectra for the tapered SM1500 fiber, with an initial diameter of $81 \mu \mathrm{m}$, with an extension of $28 \mathrm{~mm}$, for a grating period of $365 \mu \mathrm{m}$ and $30 \mathrm{~min}$ illumination time (a), and a grating period of $370 \mu \mathrm{m}$ and $45 \mathrm{~min}$ illumination time (b).

The transmission spectra of both LPGs show a single absorption dip in the wavelength range from $1408 \mathrm{~nm}$ to $1470 \mathrm{~nm}$. The absorption dip was due to the coupling of the $\mathrm{LP}_{0,1}$ core mode to the $\mathrm{LP}_{0,1}$ cladding mode, and was consistent with the optical simulation in Section 2.2. The difference in the central wavelength of the absorption $\operatorname{dip}(1435 \mathrm{~nm}$ for $\Lambda=365 \mu \mathrm{m}$ and $1450 \mathrm{~nm}$ for $\Lambda=370 \mu \mathrm{m}$, respectively), shown in Figure 10a,b, can be explained by the different grating periods that were applied for the LPG fabrication.

The strength of the absorption dip depends on the overlap between the core mode and the associated cladding mode field distribution, as well as the refractive index modulation and length of the grating. Since the LPGs shown in Figure 10 were fabricated with different exposure times to KrF excimer laser light, the strength of the absorption dip varies $(-0.62 \mathrm{~dB}$ for $\Lambda=365 \mu \mathrm{m}$ and $-0.77 \mathrm{~dB}$ for $\Lambda=370 \mu \mathrm{m}$, respectively). In Figure 11, the growth of the absorption dip as a function of $\mathrm{KrF}$ exposure time, and thus, refractive index modulation of the fiber core, is illustrated for a grating period of $365 \mu \mathrm{m}$ and a tapered SM1500 fiber with an extension length of $28 \mathrm{~mm}$ and an initial cladding diameter of $80 \mu \mathrm{m}$. Currently, the enhancement of the strength of the absorption dip is investigated by loading the tapered SM1500 fibers with hydrogen prior to the grating inscription (to enhance the photosensitivity of the fiber), as well as by replacing the chrome mask with metal sheets (to increase the pulse energy, repetition rate of the laser pulses, as well as the illumination time of the KrF excimer laser without destroying the AM), and increasing the grating length by using a beam expander from $7 \mathrm{~mm}$ to a typical length for UV-induced gratings of $10-35 \mathrm{~mm}[4,10]$. 


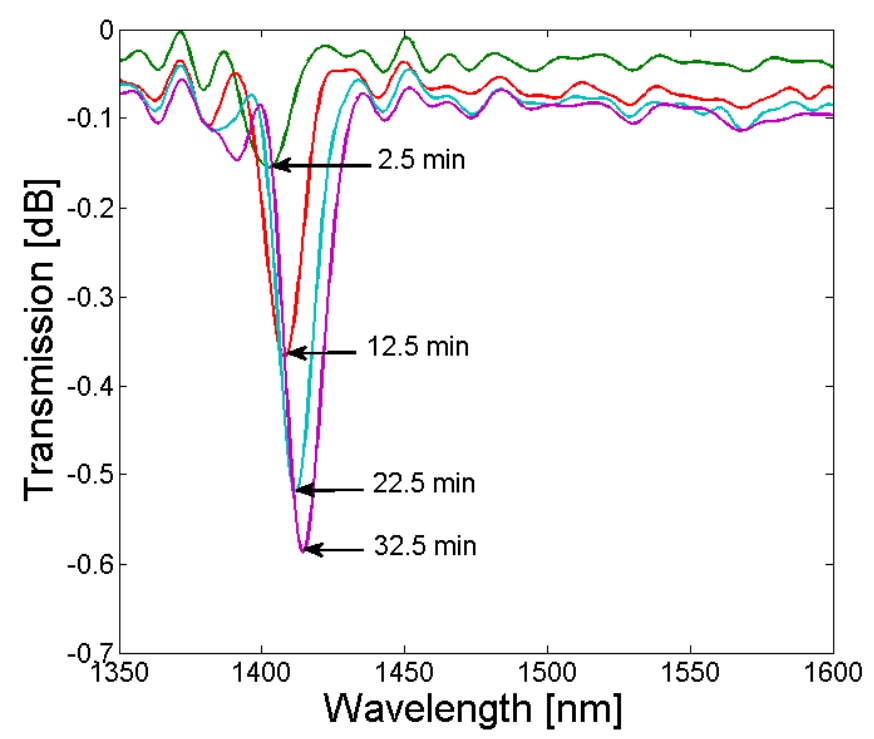

Figure 11. Gradual growth of the absorption dip with increasing UV exposure time, recorded during inscription of an LPG with a grating period of $365 \mu \mathrm{m}$ in the SM1500 fiber taper, with a starting diameter of $80 \mu \mathrm{m}$, and applying a pulse energy of $7 \mathrm{~mJ}$ and a repetition rate of $80 \mathrm{~Hz}$.

The results of the simulated and fabricated LPGs are compared in Table 1. The difference in the simulated and measured results can be explained by inaccuracies in the taper manufacturing process (diameter variations of $\pm 2.5 \%$, as mentioned in Section 3), as well as material dispersion of the SM1500, which was not taken into account during the simulation due to the lack of appropriate material models.

Table 1. Comparison between the simulated and corresponding experimental LPGs.

\begin{tabular}{ccc}
\hline Parameter & Simulation & Experiment \\
\hline initial $\mathrm{d}_{\mathrm{Cr}}(\mu \mathrm{m})$ & 4 & 4 \\
initial $\mathrm{d}_{\mathrm{Cl}}(\mu \mathrm{m})$ & 80 & 81 \\
Extension $(\mathrm{mm})$ & 28 & 28 \\
$\Lambda(\mu \mathrm{m})$ & 370 & 370 \\
$\mathrm{~L}_{\mathrm{LPG}}(\mathrm{mm})$ & 8 & 7 \\
$\delta \mathrm{n}$ & $1 \times 10^{-3}$ & $<1 \times 10^{-3}$ \\
Attenuation $(\mathrm{dB})$ & -3.26 & -0.77 \\
$\lambda_{\text {central }}(\mathrm{nm})$ & 1436 & 1449 \\
\hline
\end{tabular}

\section{Response to External Refractive Index Changes}

The sensitivity of the fabricated LPGs to refractive index changes in the surroundings was investigated by measuring the transmission spectrum (OSA, Thorlabs SLS201L/M and Ando AQ6317B) and applying different refractive index matching oils (Cargille Laboratories, Inc., Cedar Grove, NJ, USA). After each refractive index measurement, the tapered fiber containing the LPG was carefully cleaned using isopropanol. The responses of the central wavelength and amplitude of the LPG absorption dips are shown in Figures 12 and 13. Moreover, for the experiments, an etched SM1500 fiber with an initial cladding diameter of $\mathrm{d}_{\mathrm{Cl}}=80 \mu \mathrm{m}$ was tapered, to a final extension length of $28 \mathrm{~mm}$ containing a grating with a grating period of $\Lambda=365 \mu \mathrm{m}$ and a length of $\mathrm{L}_{\mathrm{LPG}}=7 \mathrm{~mm}$. From Figure 12, it follows that in the range between $n_{s}=1$ and $n_{s}=1.43$, almost no change in the amplitude of the transmission loss dip was measured. However, when the refractive index of the surroundings approached or was equal to the cladding, the refractive index of the amplitude of the transmission dip decreased, and it increased again for refractive index values of the surroundings $n_{s}>1.46$. The central wavelength of the LPG absorption dip shifts towards the blue wavelength range with an increasing 
refractive index of the surrounding medium between $n_{s}=1$ and $n_{s}=1.46$, as can be seen in Figure 13 . For refractive indices of the surrounding $n_{s}>1.46$, the central wavelength shifts back towards the red wavelength range, and remains constant for refractive index values $n_{s}>1.5$. The obtained response of the amplitude and central wavelength of the LPG absorption dip to different refractive index values of the surrounding medium agrees well with LPGs that have been fabricated using conventional techniques (e.g., [5]).

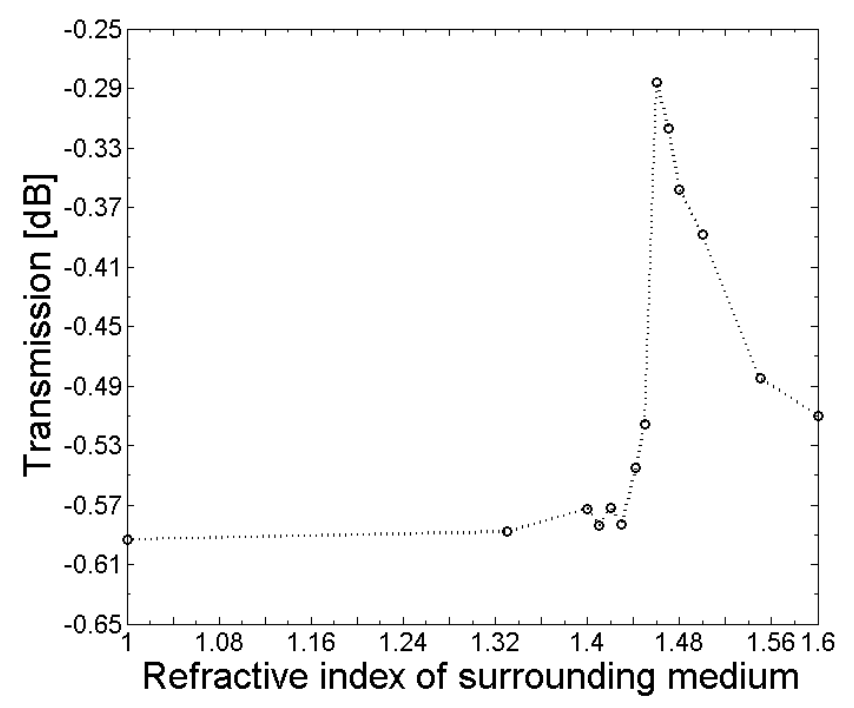

Figure 12. Dependence of the amplitude of the LPG transmission loss dip on the refractive index of the fiber surroundings.

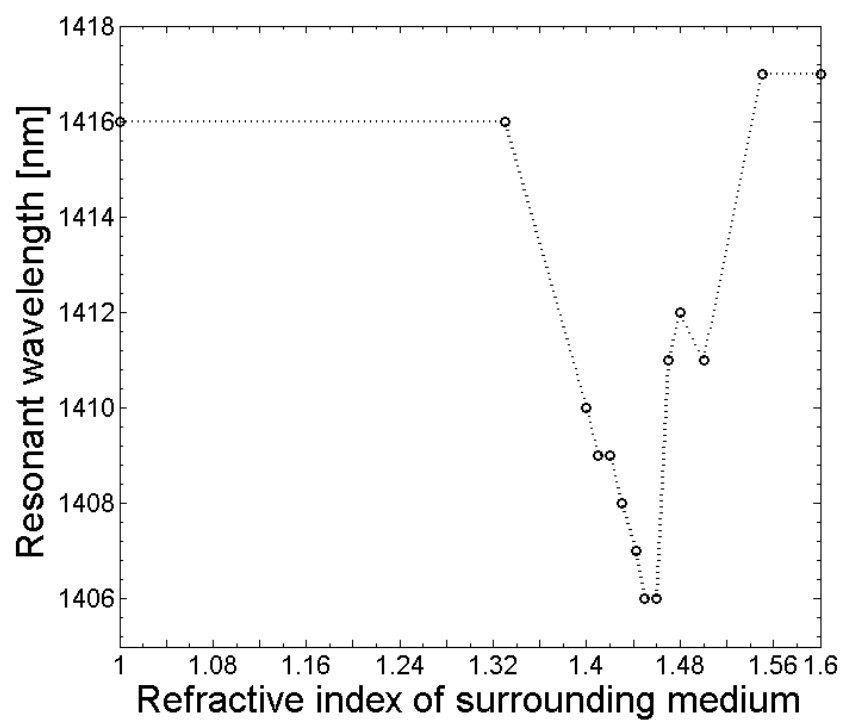

Figure 13. Dependence of the resonant LPG wavelength on the refractive index of the fiber surroundings.

\section{Conclusions}

In this study, a novel method to inscribe UV-induced LPGs into highly Ge-doped, single-mode fibers was presented. SM1500 fibers from Fibercore were used for inscription of the LPGs, and a single resonance loss dip with up to $0.77 \mathrm{~dB}$ attenuation in the wavelength range between 1400-1470 $\mathrm{nm}$ was achieved by using grating periods of $365 \mu \mathrm{m}$ and $370 \mu \mathrm{m}$, respectively, and a grating length of $7 \mathrm{~mm}$. Before the LPG was inscribed, the fiber was subject to an etching and tapering process to reduce 
core and cladding diameter, and thus, to optimize the effective refractive indices of the fundamental core mode and cladding modes, as well as the corresponding coupling coefficients. The fabrication of the LPGs was realized by applying the relatively simple and low-cost amplitude mask technique and a pulsed $\mathrm{KrF}$ excimer laser. Since highly Ge-doped, single-mode fibers offer superior features such as low-bending sensitivity and intrinsic photosensitivity compared to other fibers, the novel LPG device could be advantageous in fiber optic sensing applications, such as functionalized textile structures that are applied for SHM applications. The response of the amplitude and central wavelength of the absorption dip of the fabricated LPG spectrum is consistent with LPGs that have been fabricated with conventional fibers. Moreover, the method presented can be beneficial for telecommunication applications or the fabrication of grating assisted mode-selective fiber couplers [29].

Author Contributions: Conceptualization, S.S., K.B., B.R. and L.O.; Methodology, S.S. and K.B.; Software, S.S., K.B and Y.Z.; Validation, S.S., K.B., S.B. and F.W.; Formal Analysis, S.S., K.B. and S.B.; Investigation, S.S., K.B., Y.Z., and S.B.; Resources, J.N., B.R. and L.O.; Data Curation, S.S., K.B., Y.Z., S.B., F.W. and M.S.; Writing-Original Draft Preparation, S.S., K.B. and B.R.; Writing-Review \& Editing, S.S., K.B., M.S. and B.R.; Visualization, S.S.; Supervision, J.N., B.R. and L.O.; Project Administration, K.B. and M.S.; Funding Acquisition, J.N., B.R. and L.O.

Funding: The publication of this article was funded by the Open Access fund of Leibniz Universität Hannover.

Acknowledgments: The authors acknowledge support of the Deutsche Forschungsgemeinschaft (DFG) within Grant Number OV 36/31-1.

Conflicts of Interest: The authors declare no conflict of interest.

\section{References}

1. Liu, Y.; Zhang, L.; Williams, J.A.R.; Bennion, I. Optical Bend Sensor Based on Measurement of Resonance Mode Splitting of Long-Period Fiber Grating. IEEE Photonics Technol. Lett. 2000, 12, 531-533. [CrossRef]

2. Duan, J.; Xie, Z.; Wang, C.; Zhou, J.; Li, H.; Luo, Z.; Chu, D.; Sun, X. Torsion sensing characteristics of long period fiber gratings fabricated by femtosecond laser in optical fiber. Opt. Laser Technol. 2016, 83, 94-98. [CrossRef]

3. Kim, Y.J.; Paek, U.C.; Lee, B.H. Measurement of refractive-index variation with temperature by use of long-period fiber gratings. Opt. Lett. 2002, 27, 1297-1299. [CrossRef] [PubMed]

4. Smietana, M.; Bock, W.J.; Mikulic, P.; Chen, J. Pressure Sensing in High-Refractive-Index Liquids Using Long-Period Gratings Nanocoated with Silicon Nitride. Sensors 2010, 10, 11301-11310. [CrossRef] [PubMed]

5. Heather, P.J.; Kersey, A.D.; Bucholtz, F. Analysis of the Response of Long Period Fiber Gratings to External Index of Refraction. J. Lightwave Technol. 1998, 16, 1606-1612.

6. Chong, J.H.; Shum, P.; Haryono, H.; Yohana, A.; Rao, M.K.; Lu, C.; Zhu, Y. Measurements of refractive index sensitivity using long-period grating refractometer. Opt. Commun. 2003, 229, 65-69. [CrossRef]

7. Janczuk-Richter, M.; Dominik, M.; Rozniecka, E.; Koba, M.; Mikulic, P.; Bock, W.J.; Los, M.; Smietana, M.; Niedziolka-Jönsson, J. Long-period fiber grating sensor for detection of viruses. Sens. Actuators B Chem. 2017, 250, 32-38. [CrossRef]

8. Consales, M.; Quero, G.; Sansone, L.; Borriello, A.; Giordano, M.; Venturelli, A.; Costi, M.P.; Santucci, M.; Cusano, A. Long Period Fiber Grating Biosensor for the Detection of Drug Resistant Bacteria: The “OPTObacteria" Project. In Proceedings of the 2014 Third Mediterranean Photonics Conference, Trani, Italy, 7-9 May 2014.

9. Coelho, J.M.P.; Silva, C.; Nespereira, M.; Abreu, M.; Rebordão, J.M. Writing of Long Period Fiber Gratings Using $\mathrm{CO}_{2}$ Laser Radiation. Adv. Opt. Fiber Technol. Fundam. Opt. Phenom. Appl. 2015, 287-314. [CrossRef]

10. Nespereira, M.; Coelho, J.M.P.; Abreu, M.; Rebordao, J.M. Ultrashort Long-Period Fiber Grating Sensors Inscribed on a Single Mode Fiber Using $\mathrm{CO}_{2}$ Laser Radiation. Hindawi J. Sens. 2017, 1-9. [CrossRef]

11. Kalachev, A.I.; Nikogosyan, D.N.; Brambilla, G. Long-Period Fiber Grating Fabrication by High-Intensity Femtosecond Pulses at 211 nm. J. Lightwave Technol. 2005, 23, 2568-2578. [CrossRef]

12. Kalachev, A.I.; Pureur, V.; Nikogosyan, D.N. Investigation of long-period fiber gratings induced by high-intensity femtosecond UV laser pulses. Opt. Commun. 2005, 246, 107-115. [CrossRef]

13. Hwang, I.K.; Yun, S.H.; Kim, B.Y. Long-period fiber gratings based on periodic microbends. Opt. Lett. 1999, 24, 1263-1265. [CrossRef] [PubMed] 
14. Nam, S.H.; Zhan, C.; Lee, J.; Hahn, C.; Reichard, K.; Ruffin, P.; Deng, K.-L.; Yin, S. Bend-insensitive ultra short long-period gratings by the electric arc method and their applications to harsh environment sensing and communication. Opt. Express 2005, 13, 731-737. [CrossRef] [PubMed]

15. Hubert, G.; Malki, A. Characterizations at very high temperature of electric arc-induced long-period fiber gratings. Opt. Commun. 2002, 208, 329-335. [CrossRef]

16. Vengsarka, S.; Kosinsk, A. Splicer-based long-period fiber gratings. In Proceedings of the Optical Fiber Communication Conference and Exhibit, Technical Digest Conference: Technical Digest, San Jose, CA, USA, 22-27 February 1998; pp. 278-279.

17. Fujimaki, M.; Ohki, Y.; Brebner, J.L.; Roorda, S. Fabrication of long-period optical fiber gratings by use of ion implantation. Opt. Lett. 2000, 25, 88-89. [CrossRef] [PubMed]

18. Zhou, K.; Liu, H.; Hu, X. Tuning the resonant wavelength of long period fiber gratings by etching the fiber's cladding. Opt. Commun. 2001, 197, 295-299. [CrossRef]

19. Vengsarkar, A.M.; Lemaire, P.J.; Bhatia, V.; Erdogan, T.; Sipe, J.E. Long-Period Fiber Gratings as Band-Rejection Filters. J. Lightwave Technol. 1996, 14, 58-65. [CrossRef]

20. Bhatia, V.; Vengsarkar, A.M. Optical fiber long-period grating sensors. Opt. Lett. 1996, 21, 692-694. [CrossRef] [PubMed]

21. Zhang, L.; Liu, Y.; Everall, L.; Williams, J.A.R.; Bennion, I. Design and Realization of Long-Period Grating Devices in Conventional and High Fibers and Their Novel Applications as Fiber-Optic Load Sensors. IEEE J. Sel. Top. Quantum Electron. 1999, 5, 1373-1378. [CrossRef]

22. Mizunami, T.; Fukuda, T.; Hayashi, A. Fabrication and characterization of long-period-grating temperature sensors using Ge-B-co-doped photosensitive fibre and single-mode fibre. Meas. Sci. Technol. 2004, 15, 1467-1473. [CrossRef]

23. Lu, W.; Lu, L.; Feng, F.; Shi, J. Low-cost amplitude mask for long-period grating fabrication. Optik 2014, 125, 3462-3464. [CrossRef]

24. Regan, B.J.O.; Nikogosyan, D.N. Femtosecond UV long-period fibre grating fabrication with amplitude mask technique. Opt. Commun. 2011, 284, 5650-5654. [CrossRef]

25. Patrick, H.J.; Askins, C.G.; Mc Elhanon, R.W.; Friebele, E.J. Amplitude mask pattern on an excimer laser mirror for high intensity writing of long period fiber gratings. Electron. Lett. 1997, 33, 1408-1413. [CrossRef]

26. James, S.W.; Tatam, R.P. Optical Fibre Long-Period Grating Sensors: Characteristics and Application. Meas. Sci. Technol. 2003, 14, 49-61. [CrossRef]

27. Bremer, K.; Weigand, F.; Zheng, Y.; Alwis, L.S.; Helbig, R.; Roth, B. Structural Health Monitoring Using Textile Reinforcement Structures with Integrated Optical Fiber Sensor. Sensors 2017, 17, 345. [CrossRef] [PubMed]

28. Bruns, W.K.; Abebe, M.; Vullarruel, V.A. Parabolic model for shape of fiber taper. Appl. Opt. 1985, 24, 2753-2755. [CrossRef] [PubMed]

29. Bremer, K.; Lochmann, S.; Roth, B. Grating assisted optical waveguide coupler to excite individual modes of a multi-mode waveguide. Opt. Commun. 2015, 356, 560-564. [CrossRef]

(C) 2018 by the authors. Licensee MDPI, Basel, Switzerland. This article is an open access article distributed under the terms and conditions of the Creative Commons Attribution (CC BY) license (http://creativecommons.org/licenses/by/4.0/). 